\title{
Musikbezogene Quellen aus der Korrespondenz zwischen Rom und dem Wiener Kaiserhof. Neues \\ zu Antimo Liberati, Alessandro Stradella und anderen italienischen Musikern und Musikerinnen des Barock
}

\section{Music-Related Sources from the Correspondence between Rome and the Viennese Court. New Material Concerning Antimo Liberati, Alessandro Stradella and other Italian Musicians from the Baroque Era}

Marko Deisinger / deisinger@mdw.ac.at

University of Music and Performing Arts Vienna, AT

\begin{abstract}
This article unearths hitherto unknown source material dating from 1640-1688, which is preserved as part of the imperial court's correspondence at the National Archives of Austria in Vienna. The discovery of these sources not only offers further evidence of the music-historical relationships between Italy and the Viennese Habsburg court, but it also provides revealing biographical details of Italian musicians, among whom are famous composers as well as singers such as Vincenzo Oliviciano, Angelica Voglia and Giovanni Francesco Grossi, known as Siface.
\end{abstract}

\section{Keywords}

17th-century music, Italian musicians, Habsburg Court in Vienna, Ferdinand III, Leopold I, cultural transfer 
Im Zentrum des vorliegenden Beitrags stehen in der Fachliteratur bisher unbekannte Quellen zu italienischen Musikern und Musikerinnen aus dem Zeitalter des Barock. Das aus der Korrespondenz zwischen Rom und dem Wiener Kaiserhof stammende Quellenmaterial befindet sich heute im Österreichischen Staatsarchiv. Es zeugt einerseits von den intensiven musikkulturellen Beziehungen, die die barocken Habsburgerherrscher mit Italien pflegten, und liefert andererseits aufschlussreiche Details zu den Biografien der thematisierten Musiker und Musikerinnen. Im Folgenden werden die aufgefundenen Quellen in ihrem geschichtlichen Kontext präsentiert und besprochen, wobei die eine oder andere biografische Lücke geschlossen werden soll. Das untersuchte Material umfasst die Jahre 1640 bis 1689. Die Besprechung erfolgt weitgehend chronologisch und berücksichtigt nur Quellen, in denen Musiker oder Musikerinnen namentlich genannt werden.

Zunächst findet sich in einem vom 5. Juni 1640 datierten Schreiben an Kaiser Ferdinand III. der Name des Musikers Giacinto Cornacchioli, der in diesem Jahr in Italien unterwegs war, um Musiker für den Erzherzog Leopold Wilhelm anzuwerben. ${ }^{1}$ Das Schreiben stammt vom kaiserlichen Gesandten in Rom, Scipione Gonzaga, Fürst von Bozzolo und Herzog von Sabbioneta, und wurde in Bozzolo verfasst, wo sich Gonzaga seit Anfang April 1640 aufhielt, um häusliche Angelegenheiten zu erledigen. ${ }^{2}$ In seinem Schreiben meint Gonzaga, dass er Cornacchioli in seiner Mission nicht wie beauftragt assistieren könne, da ihn der Brief des Kaisers vom 8. März erst vor drei Tagen erreicht habe. Er werde dies aber nach seiner Rückkehr in Rom nachholen, sofern er dort ohne Verspätung ankommen sollte (Anhang I). Um welche Aufgabe es sich dabei konkret handelte, wird nicht erwähnt. Jedoch ist anzunehmen, dass sie im Zusammenhang mit der Rekrutierung von Musikern stand.

Gonzagas Nachfolger im Amt des kaiserlichen Gesandten in Rom war Herzog Federico Savelli. ${ }^{3}$ Dieser schreibt am 28. April 1646 an den Kaiser, dass er wie von ihm beauftragt mit dem Papst über den ehemals in der päpstlichen Kapelle beschäftigten Musiker Pietro Giorgio Piccolini gesprochen habe. Außerdem habe er dem Papst die Bittschrift vorgelegt und dessen Bescheid abgewartet, nachdem er hatte feststellen müssen, dass die Meinung des Papstes über den Musiker keine besonders gute sei, weil sich dieser in der Vergangenheit schlecht benommen habe. Der Bescheid besage, dass die Sache zu gegebener Zeit wieder in Erinnerung gerufen werde (Anhang II).

1 Vgl. ANTONICEK, Theophil. „Pigliar musici dall' Italia“. Ein Agent des Erzherzogs Leopold Wilhelm auf der Suche nach italienischen Musikern: Giacinto Cornacchioli. Studien zur Musikwissenschaft, 2017, vol. 59 , S. 8.

2 Vgl. BECKER, Rotraud. Die Neubesetzung der kaiserlichen Gesandtschaft in Rom im Jahr 1634. Italienische Fürsten als Gesandte des Heiligen Römischen Reiches. Quellen und Forschungen aus italienischen Archiven und Bibliotheken, 2014, vol. 94, S. 247.

3 Vgl. ibid., S. 248-250 und FOSI, Irene. La famiglia Savelli e la rappresentanza imperiale a Roma nella prima metà del Seicento. In Kaiserhof - Papsthof (16.-18. Jahrhundert). Richard Bösel - Grete Klingenstein - Alexander Koller (Hg.). Wien: Verlag der Österreichischen Akademie der Wissenschaften, 2006, S. 75. Zu Savellis' Rolle im kulturellen Austausch zwischen Rom und Wien siehe MAZZETTI DI PIETRALATA, Cecilia. Paolo e Federico Savelli, ambasciatori dell'imperatore. Scambi artistici e musicali tra Roma e Vienna nella prima metà del Seicento. In La Dinastía de los Austria. Las relaciones entre la Monarquía Católica y el Imperio, vol. 3. José Martínez Millán - Rubén González Cuerva (Hg.). Madrid: Ediciones Polifemo, 2011, S. 1837-1866. 
Bei der erwähnten Bittschrift handelt es sich offenbar um ein beim Kaiser eingereichtes Gesuch Piccolinis um eine Empfehlung für die Bewerbung um einen Posten in der päpstlichen Kapelle. Ein Blick in Piccolinis Biografie verrät uns, dass der Musiker kein zweites Mal in diese Kapelle aufgenommen wurde. Der in Vercelli geborene Basssänger Piccolini, oder Picciolini, ${ }^{4}$ diente zunächst am polnischen Königshof, ${ }^{5}$ ehe er 1618 in den Dienst des Papstes trat. 1629 wurde er aus Rom verbannt. Von Anfang April bis Ende September 1637 wirkte er in der Wiener Hofkapelle Ferdinands III. 1640 war Piccolini Mitglied der Hofmusik des Herzogs von Savoyen in Turin, und 1645 ist er schließlich wieder in Rom nachweisbar. ${ }^{6}$

Während Piccolini in Wien mit einer Ehrenkette geschmückt aus dem kaiserlichen Dienst entlassen wurde, hatte er in Rom wiederholt Probleme verursacht, worauf auch seine Verbannung aus der Stadt zurückzuführen ist. Beispielsweise weigerte er sich in der Fastenzeit von 1625 einen Part zu singen, den ihm der Kapellmeister zugeteilt hatte. ${ }^{7}$ Darüber hinaus musste er wegen Überschuldung eine gerichtlich angeordnete Gehaltspfändung hinnehmen. ${ }^{8}$ Wie aus Savellis Schreiben ersichtlich, hatte man in Rom das ehrlose Verhalten Piccolinis noch viele Jahre später in Erinnerung.

Ein weiteres Schreiben Savellis, in dem ein Musiker genannt wird, ist vom 18. September 1649 datiert. In diesem berichtet Savelli dem Kaiser, dass er von seinem Kammermusiker Marc'Antonio Ferri kürzlich das Schreiben vom 15. Juli dieses Jahres erhielt, das der Kaiser zugunsten des Musikers verfasst hatte, und dass er diesen bestmöglich unterstützen werde (Anhang III). Das von Savelli erwähnte kaiserliche Schreiben hat sich in einem römischen Archiv erhalten. In lateinischer Sprache abgefasst, fordert es den Botschafter dazu auf, Ferri unter seinen Schutz zu stellen. Zudem wird der Leser darin in Kenntnis gesetzt, dass Ferri nach Italien gereist war, um gewisse Angelegenheiten zu erledigen. $^{9}$

Marc'Antonio Ferri (Ferro) war von 1642 bis 1652 Hoflautenist Ferdinands III. und unterrichtete dessen Sohn Ferdinand IV. Für seine Verdienste wurde er vom Kaiser in den Adelsstand erhoben. 1649 ließ er in Venedig als sein Opus 1 eine Sammlung von Kirchensonaten (Sonate a due, tre, E quattro) drucken, die er seinem Dienstherrn

4 Vgl. FERRAROTTI, Mario. Pietro Giorgio Picciolini: Basso vercellese. Vercelli: De Marchi, 1946.

5 Vgl. PRZYBYSZEWSKA-JARMIŃSKA, Barbara. Muzyczne kontakty dworu królewskiego polskich Wazów z Rzymem w świetle dawniejszych i nowych badań. De Musica / Diagonali, 2012, vol. 1, S. 15, 28. PRZYBYSZEWSKA-JARMIŃSKA, Barbara. The Careers of Italian Musicians Employed by the Polish Vasa Kings (1587-1668). Musicology Today, 2009, vol. 6, S. 31.

6 Vgl. Piccolini, Pietro Giorgio. In Großes Sängerlexikon, vol. 5. München: Saur, 2003, S. 3657.

7 Vgl. ROSTIROLLA, Giancarlo. Celebrazioni ed eventi musicali nella Cappella Pontificia durante l'Anno Santo 1625: Dal Diario redatto dal compositore e cantore perugino Francesco Severi, con l'edizione integrale di esso. In "Vanitatis fuga, aeternitatis amor«. Wolfgang Witzenmann zum 65. Geburtstag. Laaber: Laaber-Verlag, 2005, S. 162, 184.

8 Vgl. DIXON, Graham. Lorenzo Ratti (1589/90-1630): "Exemplum Virtutum". Note d'Archivio per la Storia Musicale, 1984, vol. 2, S. 16.

9 Archivio di Stato di Roma, Archivio Sforza-Cesarini, Parte prima (etichetta rettangolare), busta 14 (Ferdinand III. an Federico Savelli, Wien, 15. Juli 1649), zit. nach BORCHIA, Matteo. Gli agenti delle corti tedesche a Roma nel XVIII secolo. Dissertation. Rom 2010-2011, S. 45f. [cit. 2018-01-18]. URL: <http://padis.uniroma1. it/bitstream/10805/1728/1/Tesi_Borchia.pdf> 
widmete. ${ }^{10}$ Die Annahme liegt nahe, dass seine Reise nach Italien mit der Drucklegung und dem Erscheinen dieser Sonaten im Zusammenhang stand. In Rom könnten seine Kompositionen zu Werbe- und Repräsentationszwecken zur Aufführung gelangt sein. ${ }^{11}$

Die nächsten vier Dokumente, die im Folgenden besprochen werden, stammen alle aus dem Jahre 1655 und betreffen einen Musiker, der ebenso wie Cornacchioli, Piccolini und Ferri eine Zeitlang im Dienst der Habsburger stand, nämlich den vor allem als Komponist in die Musikgeschichte eingegangenen Antimo Liberati. Liberati war in seiner Jugendzeit nachweisbar Musiker der verwitweten Kaiserin Eleonora I. und diente eigenen Angaben zufolge Erzherzog Leopold Wilhelm und Kaiser Ferdinand III. in Wien. ${ }^{12}$ Nach seiner Rückkehr nach Italien bewarb er sich 1646, 1648 und 1652 um den Posten eines Sängers in der päpstlichen Kapelle, musste sich aber im Laufe der Aufnahmewettbewerbe stets geschlagen geben. Erst im November 1661 bestand er als Altist die Prüfung zur Aufnahme ins päpstliche Sängerkollegium. In dem von ihm verfassten Diarienband der Cappella Sistina von 1670 vermerkte er, dass er vier Mal an der Aufnahmeprüfung gescheitert war. ${ }^{13}$

Dass sich Liberati vor 1661 tatsächlich ein viertes Mal um einen vakanten Sängerposten in der päpstlichen Kapelle bemüht hatte, belegt ein von ihm bei Ferdinand III. am 11. Februar 1655 eingereichtes Gesuch. Als ehemaliger Musiker des Hauses Habsburg bat er darin den Kaiser entweder Kardinal Ernst Adalbert von Harrach oder Kardinal Friedrich von Hessen-Darmstadt schriftlich dazu aufzufordern, beim neuen Papst ${ }^{14} \mathrm{zu}$ seinem Gunsten zu intervenieren mit dem Ziel, die frei gewordene Stelle eines Altisten oder einen Posten als Zusatzmusiker zu bekommen. Im Gegenzug würde er als ständiger Diener des Kaisers in Rom jeglichen Auftrag aus Wien entgegennehmen und Informationen über jene Musiker liefern, die der Kaiser in seine Kapelle aufzunehmen beabsichtige. Außerdem geht aus dem Gesuch hervor, dass sich Liberati damit erhoffte, den üblichen Aufnahmewettbewerb zu umgehen (Anhang IV).

10 Vgl. BOISITS, Barbara. Ferro, Marco Antonio. In Oesterreichisches Musiklexikon (=OeMl), online. [cit. 201801-18]. URL: 〈http://www.musiklexikon.ac.at/ml/musik_F/Ferro_Marco.xml〉. SEIFERT, Herbert. 16191792: „Die Kaiserlichen Hofkapellen“. In SEIFERT, Herbert. Texte zur Musikdramatik im 17. und 18. Jahrhundert. Aufsätze und Vorträge. Matthias J. Pernerstorfer (Hg.). Wien: Hollitzer, 2014, S. 594.

11 Ferri wurde 1652 aus der kaiserlichen Hofkapelle entlassen, da er in Ungnade gefallen war. Jedoch nahm ihn Leopold I. 1658 wieder auf. Dass sich Ferri 1656 und 1657 in Rom aufhielt, geht aus Gehaltslisten hervor, in denen Musiker verzeichnet sind, die vorübergehend in der Basilika S. Maria Maggiore Dienste leisteten („Musici forastieri“). Bei dem darin verzeichneten „S.r Ferro Leuto“ dürfte es sich wohl um Marc' Antonio Ferri handeln. Siehe CILIBERTI, Galliano. Antonio Maria Abbatini e la musica del suo tempo (1595-1679). Documenti per una ricostruzione bio-bibliografica. Perugia: Gestisa, 1986, S. 237-238.

12 Vgl. SEIFERT, Herbert. Liberati, Antimo. In OeMl, online. [cit. 2018-01-18]. URL: http://musiklexikon. ac.at/ml/musik_L/Liberati_Antimo.xml. MORELLI, Arnaldo. Liberati, Antimo. In Dizionario Biografico degli Italiani (= DBI), 2005, vol. 65, online. [cit. 2018-01-18]. URL: http://www.treccani.it/enciclopedia/antimo-liberati_(Dizionario-Biografico).

13 Vgl. RAMBOTTI, Fiorella. "La musica è una mera opinione e di questa non si può dar certezza veruna". Antimo Liberati e il suo Diario sistino con una riproduzione della Lettera a Ovidio Persapegi. Perugia: Morlacchi, 2008, S. 19-20. HEYINK, Rainer. Fest und Musik als Mittel kaiserlicher Machtpolitik. Das Haus Habsburg und die deutsche Nationalkirche in Rom S. Maria dell'Anima. Tutzing: Schneider, 2010, S. 73.

14 Der Stuhl Petri war zu diesem Zeitpunkt vakant. Innozenz X. starb am 7. Januar 1655. Sein Nachfolger Alexander VII. regierte ab 7. April 1655. 
Dass dem Gesuch stattgegeben wurde, belegt eine erhalten gebliebene Abschrift eines in lateinischer Sprache verfassten Schreibens an Kardinal Harrach vom 17. Februar 1655 (Anhang V). ${ }^{15}$ Trotz der kaiserlichen Empfehlung blieb Liberati in seinem Bestreben erfolglos, gab sich aber noch nicht geschlagen und suchte wenige Monate später, am 11. Juli 1655, beim Kaiser erneut um eine Empfehlung an, die ihm zu einem außerplanmäßigen Posten in der päpstlichen Kapelle verhelfen sollte. Diesmal bat er, die Empfehlung durch Kardinal Giovan Carlo de' Medici an den Papst gelangen zu lassen (Anhang VI). Wiederum entsprach der Kaiser dem Gesuch und richtete am 2. August 1655 an den Kardinal ein lateinisches Schreiben, von dem sich ein Entwurf erhalten hat (Anhang VII). Wie wir aus Liberatis Biografie wissen, führte auch dieser Versuch zu keinem Erfolg.

Im Folgenden kommen Quellen zur Besprechung, die alle aus einem größeren, in sich geschlossenen Archivbestand einheitlicher Entstehung stammen. Es handelt sich dabei um die Berichte des habsburgischen Kardinalprotektors Carlo Pio di Savoia, ${ }^{16}$ der diese von 1676 bis zu seinem Tod im Februar 1689 wöchentlich aus Rom an Kaiser Leopold I. schrieb. Seine Berichte sind in der Regel zweigeteilt. Sie bestehen aus einem allgemein gehaltenen Nachrichtenteil, der von einem professionellen Schreiber verfertigt wurde, und einem mit „Riservato“ betitelten Abschnitt, in dem der Kardinal aktuelle Geschehnisse näher kommentiert und selbst Erlebtes darlegt. Thematisiert werden nicht nur politische Angelegenheiten, sondern auch Ereignisse aus dem kulturellen und gesellschaftlichen Leben der Stadt. ${ }^{17}$

So berichtet Pio am 24. Oktober 1676 von einer skandalösen Affäre um eine versuchte Kuppelei, in der der Komponist Alessandro Stradella verwickelt war. Dieser Vorfall wurde in der Fachliteratur bereits mehrmals dargestellt. ${ }^{18}$ Die bisher bekannten Informationen darüber sind teils sehr vage, da sie aus Berichten von Personen stammen, die nicht unmittelbar am Geschehen beteiligt waren. Zudem beschränkt sich die Anzahl dieser Berichte auf drei Stück. Diesen zufolge ließ sich Stradella von einer Frau 10.000 Scudi bezahlen, um sie mit einem reichen Verwandten des einflussreichen Kardinals und päpstlichen Staatssekretärs Alderano Cibo zu verkuppeln. Zusammen mit seinem Komplizen, dem päpstlichen Gesangskastraten Giovanni Battista Vulpio, arrangierte Stradella eine Zusammenkunft mit Musik und Gesang, in deren Verlauf die beiden Musiker den Verwandten des Kardinals betrunken machten und anschließend einen Priester holten, der den betrunkenen Mann mit der Frau traute. Nachdem Kardinal Cibo davon erfahren

15 Die Archiveinheit, in der sich diese Abschrift befindet, enthält auch ein lateinisches Dokument über den Musiker Pietro Santi vom 28. Januar 1677.

16 Carlo Pio di Savoia (1622-1689) war ab 1673 Kardinalprotektor der Österreichischen Erbländer und von 1682 bis zu seinem Tod Kardinalprotektor der Deutschen Nation.

17 Pios Berichte sind auch Untersuchungsgegenstand folgender Studie: NEVEU, Bruno. Episcopus et Princeps Urbis: Innocent XI réformateur de Rome d'après des documents inédits (1676-1689). In Römische Kurie. Kirchliche Finanzen. Vatikanische Archive. Studien zu Ehren von Hermann Hoberg, vol. 2. Erwin Gatz (Hg.). Rom: Università Gregoriana Editrice, 1979, S. 597-633.

18 Vgl. GIANTURCO, Carolyn. Stradella. «uomo di gran grido». Pisa: Edizioni ETS, 2007, S. 47-48, 269. SCHRAMMEK, Bernhard. Stradella, Alessandro. In Die Musik in Geschichte und Gegenwart. Allgemeine Enzyklopädie der Musik, Personenteil, vol. 16. Ludwig Finscher (Hg.). 2. Ed., Kassel \& Stuttgart 2006, Sp. 1572. 
hatte, ließ er die Frau in ein Kloster stecken und machte Vulpio den Prozess. Stradella entging seiner Verhaftung durch Flucht, die ihn schließlich nach Venedig führte.

Aus Pios Bericht können bislang unbekannte Einzelheiten zu dem Skandal entnommen werden, z. B. der Name des Opfers der Kuppelei („Innocenzio Cibo“) oder der Standort des Klosters („Conservatorio di Monte Citorio“) ${ }^{19}$ in dem die als „la Pescatora“ bekannte Frau den Rest ihres Lebens verbringen musste. ${ }^{20}$ Darüber hinaus geht aus Pios Bericht hervor, dass sich der Vorfall in den Zimmern des Kastraten Vulpio ereignete, der damals gerade im Dienst des Herzogs von Bracciano, Flavio Orsini, stand und in dessen Haus untergebracht war (Anhang VIII.1). ${ }^{21}$

In den Nachrichten, die Pio dem Kaiser übermittelte, kommt neben Stradella noch ein zweiter Komponist vor. Die Rede ist von Antonio Masini, über den gemeldet wird, dass im Februar 1678 Aufführungen einer „comedia“ von ihm im Hause des „Monsù Ridò" stattfanden und dass sich unter den Anwesenden die Königin Christina von Schweden befand (Anhang VIII.3). Masini stand zu diesem Zeitpunkt als Kammermusiker im Dienst der schwedischen Königin und hatte zudem den Kapellmeisterposten an S. Pietro im Vatikan inne. Bei dem aufgeführten Werk handelt es sich möglicherweise um Il Narciso, eine „commedia“ Masinis, die durch eine römische Quelle aus dem Jahre 1678 belegt ist. ${ }^{22}$

In Pios Berichten über das römische Musikleben werden aufgeführte Werke, deren Autoren sowie ausführende Musiker nur in seltenen Fällen namentlich erwähnt. Letztere sind ausschließlich Sänger und Sängerinnen, die mit ihrer Virtuosität im Rampenlicht der Öffentlichkeit standen. Am 12. August 1684 übersandte Pio die Nachricht von einer Serenata, die zwei Tage zuvor bei der Königin von Schweden mit vielen Instrumenten aufgeführt wurde und in der „Ballarino“, Altist des Herzogs von Mantua, sang (Anhang VIII.6). ${ }^{23}$ Der gebürtige Florentiner Francesco Ballerino, oder Ballerini, war ursprünglich Musiker des Großherzogs der Toskana, ehe er in den Dienst des mantuanischen Herzogs Ferdinando Carlo trat. Als dessen Virtuose unternahm er ab den 1680er Jahren immer wieder Gastspielreisen, die ihn in zahlreiche italienische Städte führten. 1695 ging Ballerino nach Wien, wo er am Kaiserhof zunächst als Altist und dann als Kammermusiker beschäftigt war. ${ }^{24}$

19 Im ursprünglichen Franziskanerinnen-Kloster Santa Croce a Montecitorio waren von 1672 bis 1695 die Schwestern vom Heiligen Filippo Neri (Oblate filippine) untergebracht. Siehe CAFFIERO, Marina. Il sistema dei monasteri femminili nella Roma barocca. Insediamenti territoriali, distribuzione per ordini religiosi, vecchie e nuove fondazioni. Dimensioni e problemi della ricerca storica, 2008, vol. 2. S. 78, 90, 96-97, 100.

20 Vgl. GIANTURCO, op. cit., S. 47.

21 Orsini war auch ein Gönner Stradellas. Siehe GOULET, Anne-Madeleine. Orsini, Flavio. In DBI, 2013, vol. 79, online. [cit. 2018-01-18]. URL: http://www.treccani.it/enciclopedia/flavio-orsini_(Dizionario-Biografi$\mathrm{co})$.

22 Vgl. MORELLI, Arnaldo. Masini, Antonio. In DBI, 2008, vol. 71, online. [cit. 2018-01-18]. URL: http:// www.treccani.it/enciclopedia/antonio-masini_(Dizionario-Biografico).

23 Vgl. STAFFIERI, Gloria. Colligite Fragmenta. La vita musicale romana negli «Avvisi Marescotti» (1683-1707). Rom: Libreria musicale italiana editrice, 1990, S. 60-61.

24 Vgl. SEIFERT, Herbert. Nordwärts reisende Gesangsvirtuosen aus Italien und ihr stilistisches „Gepäck“ im Seicento. In SEIFERT, Texte zur Musikdramatik, op. cit., S. 167-168. 
Über das Gastspiel eines anderen virtuosen Sängers, der ebenfalls zeitweise für den Kaiser arbeitete, erfahren wir aus einem Bericht, den Pio am 19. April 1687 nach Wien schickte. Bei dem erwähnten Sänger handelt es sich um den weitgereisten und die meiste Zeit seines Lebens im Dienst des toskanischen Großherzogs Cosimo III. de' Medici stehenden Vincenzo Oliviciano, genannt Vincenzino. ${ }^{25}$ Dem Bericht ist zu entnehmen, dass Vincenzino im Haus von Kardinal Francesco Maria de' Medici, also dem Bruder seines Dienstgebers, sang und dass sich dort viele Leute einfanden, um den Virtuosen zu hören (Anhang VIII.13). Pios Mitteilungen zufolge war Vincenzino zwei Monate später, am Abend des 16. Juni 1687, ein weiteres Mal in Rom zu hören. An diesem Tag soll er zusammen mit anderen auswärtigen Musikern an einer von der schwedischen Königin organisierten "Academia in Musica“ teilgenommen haben (Anhang VIII.15).

Weitere Gesangsvirtuosen, die in Pios Berichten Erwähnung finden, sind die beiden päpstlichen Musiker Francesco Maria Fede ${ }^{26}$ und Giovanni Ricchi. ${ }^{27}$ Bekannt gegeben werden deren Ableben und die daraus resultierenden Folgen. Während man über Fede erfährt, dass eine Darmverschlingung seinen Tod verursacht hatte und dass der aufgrund dessen frei gewordene Posten in der päpstlichen Kapelle zum Zeitpunkt der Berichterstattung immer noch vakant war, heißt es über Ricchi, dass die Stelle, die er als Kanoniker der Kirche Santa Maria ad Martyres (Pantheon) hinterlassen hatte, am Tag nach seinem Tod mit einem von Livio Odescalchis ${ }^{28}$ Sekretären nachbesetzt wurde (Anhang VIII.5 und 9).

Auch der berühmte Kastrat Giovanni Francesco Grossi, genannt Siface, taucht in Pios Berichten auf. Thematisiert wird eine durch mehrere Quellen belegte und in der Literatur wiederholt nachgezeichnete Begebenheit, wonach sich Siface 1683 in Rom weigerte, für den französischen Botschafter François Annibal II. d'Estrées zu singen. ${ }^{29}$ In Pios Bericht stellt sich die Sache wie folgt dar: Nachdem der Botschafter den Sänger um eine Darbietung gebeten hatte, antwortete dieser, dass er es satt habe, unentgeltlich aufzutreten, und warf dem Botschafter vor, ein Schmarotzer zu sein, da er ihm bisher

25 Vgl. GLÜXAM, Dagmar. Oliviciano (Olivicciani, Oliviciani, Ulivicciani), Vincento (Vincenzo, Fincenzo), gen. Vincenzino. In $O e M l$, online. [cit. 2018-01-18]. URL: http://www.musiklexikon.ac.at/ml/musik_O/Oliviciano_Vincenzo.xml.

26 Näheres über den am 1. März 1684 verstorbenen Soprankastraten siehe ROSTIROLLA, Giancarlo. Fede, Francesco Maria. In DBI, 1995, vol. 45, online. [cit. 2018-01-18]. URL: http://www.treccani.it/enciclopedia/ francesco-maria-fede_(Dizionario-Biografico). Ein Bericht über seinen Tod findet sich auch in STAFFIERI, op. cit., S. 58.

27 Ricchi starb am 16. Oktober 1685. Über den aus Lucca stammenden Tenor und Geistlichen ist wenig bekannt. In der Literatur gib es nur vereinzelt Hinweise auf seinen Werdegang, z. B. in ADAMI, Andrea. Osservazioni per ben regolare il coro de i cantori della Cappella Pontificia. Rom: Antonio de' Rossi alla Piazza di Ceri, 1711, S. 207. CILIBERTI, op. cit., S. 360, 380, 384, 388, 390, 501, 507, 512. GIANTURCO, Carolyn. Cristina di Svezia scenarista per Alessandro Stradella . In Convegno Internazionale. Cristina di Svezia e la Musica (Roma, 5-6 dicembre 1996). Rom: Accademia Nazionale dei Lincei, 1998, S. 64. MONALDINI, Sergio. L'Orto dell'Esperidi. Musici, attori e artisti nel patrocinio della famiglia Bentivoglio 1646-1685. Lucca 2001, S. XXIV, 116-117.

28 Livio Odescalchi war Neffe und Günstling des damaligen Papstes Innozenz XI.

29 Vgl. TILMOUTH, Michael - BALDWIN, Olive - WILSON, Thelma. Siface [Grossi, Giovanni Francesco ]. In The New Grove Dictionary of Music and Musicians, vol. 23. Stanley Sadie (Hg.). 2. Ed. London:

Macmillan, 2001, S. 370. 
noch nie etwas gegeben habe, wenn er für ihn sang. Der Botschafter war daraufhin so erzürnt, dass er ihm mit Bestrafung drohte und den Kardinal Francesco Maidalchini, bei dem Siface wohnte, dazu aufforderte, den Sänger zu verjagen. Schließlich begab sich Siface zuerst in den Palazzo seines Dienstgebers, des Herzogs von Modena, und verließ Gerüchten zufolge dann die Stadt (Anhang VIII.4).

Relativ oft finden sich in Pios Berichten Notizen über Sängerinnen, die alle allerdings nicht mit ihren Realnamen aufscheinen. Zunächst begegnen wir in zwei Berichten aus dem Jahre 1678 der Sängerin und Poetin Anna Rosalia Carusi, die allgemein bekannt als „la Baronessa“ im römischen Musikleben eine nicht unwichtige Rolle spielte. Sie unterhielt enge Kontakte zu einflussreichen Adeligen, die sie geschickt dazu nutzte, um ihre angesehene Stellung als Künstlerin und Kunstmäzenin auszubauen. Unter anderem organisierte sie Aufführungen von musikdramatischen Werken, zu denen auch jene im Februar 1678 mehrmals gesungene Oper zählt, von der Pio berichtete (Anhang VIII.2 und 3). ${ }^{30}$

1684 machte Pio dem Kaiser die Mitteilung, dass die Baronessa mit Hilfe der Herzogin von Bracciano ${ }^{31}$ ins Kloster Sant'Ambrogio eingetreten, dort aber auf den Wiederstand einiger Nonnen gestoßen sei, die schließlich mit Hilfe des Papstes ihren Ausschluss bewirken konnten. Laut Pio beriefen sich die Nonnen auf eine bis zu diesem Zeitpunkt nie beachtete Regel, die besagt, dass die Schwestern ihres Klosters nicht älter als 25 Jahre sein dürfen - eine Altersgrenze, die Carusi aber schon überschritten hatte (Anhang VIII.7 und 8). ${ }^{32}$

Während Carusi auf Anordnung des Papstes das Kloster verlassen musste, musste eine andere Sängerin darum kämpfen, vom Papst nicht ins Kloster gesteckt zu werden. Darüber erfahren wir aus einem Bericht, den Pio am 25. Mai 1686 verfasste. ${ }^{33}$ Bei dieser Sängerin handelt es sich um Angelica Voglia, genannt „la Giorgina“. ${ }^{34}$ Um nicht ihre Freiheit zu verlieren, stellte sich die Sängerin unter den Schutz ihrer Gönnerin, der Königin Christina von Schweden (Anhang VIII.10). Dieser Mitteilung ließ Pio im Sommer eine weitere Nachricht über die Sängerin folgen, wonach Giorgina am 24. Juli 1686 in einer gut besuchten Aufführung einer Serenata mitwirkte, die in der Residenz der schwedischen Königin stattfand (Anhang VIII.11).

Zwei weitere Notizen über Auftritte der Sängerin im Umfeld der Königin Christina finden sich in den Berichten der Folgejahre. Anfang Januar 1687 brachte Pio dem Kaiser zur Kenntnis, dass Giorgina in einer Abendunterhaltung sang, die von Orazio Del Monte, einem Angehörigen des Hofstaats der schwedischen Königin, für Marchese di

30 Vgl. TAMBURINI, Elena. La lira, la poesia, la voce e il teatro musicale del Seicento: note su alcune vicende biografiche e artistiche della baronessa Anna Rosalia Carusi. In La musica a Roma attraverso le fonti d'archivio. Atti del convegno internazionale, Roma 4-7 giugno 1992. Bianca Maria Antolini - Arnaldo Morelli - Vera Vita Spagnuolo (Hg.). Lucca: Libreria Musicale Italiana, 1994, S. 421-422, 429. D’ACCONE, Frank A. Ancora su l'opera prima di Scarlatti e la Regina. In Convegno Internazionale, op. cit., S. 80-81.

31 Flavio Orsinis zweite Ehefrau Marie-Anne de la Trémoille.

32 Vgl. NEVEU, op. cit., S. 618-619.

33 Vgl. STAFFIERI, op. cit., S. 70-71.

34 Näheres zu Voglia siehe ibid., S. 68 und MORELLI, Giorgio. Una celebre "Canterina" romana del Seicento, La Giorgina. Studi Secenteschi, 1975, vol. 16, S. 157-180. 
Solera, Sohn des sizilianischen Vizekönigs, ${ }^{35}$ veranstaltet wurde (Anhang VIII.12). Und in einem Bericht vom 10. Juli 1688 heißt es, dass Giorgina zusammen mit einer Kollegin, der Tochter des Hauptmanns Landini, in einer Serenata auftrat, deren Darbietung in der Residenz der Königin von Schweden vor einer großen Zuhörerschaft erfolgte (Anhang VIII.17). Bei der genannten zweiten Sängerin handelt es sich um Maria Landini. Diese war die illegitime Tochter von Orazio Del Monte und Stiefkind von Francesco Landini, dem Hauptmann von Christinas Leibwache. Nach dem Tod der Königin, ihrer Patronin, machte sie als Sopranistin in Oberitalien und nördlich der Alpen Karriere. Ihr letzter Wirkungsort war der Wiener Kaiserhof, wo Landini in allen Opern von Johann Joseph Fux mitwirkte. ${ }^{36}$

Neben der „Baronessa“ und der „Giorgina“ konnte in den Berichten von Kardinal Pio noch eine „Capitaniessa“ genannte Sängerin entdeckt werden, von der mitgeteilt wird, dass sie am 21. April 1687 in einer von Abate Elpidio Benedetti organisierten Festveranstaltung anlässlich der Genesung des französischen Königs sang (Anhang VIII.14). ${ }^{37}$ Diese Sängerin konnte bisher nicht identifiziert werden. Zumindest lässt sich annehmen, dass es sich bei „la Capitaniessa“ um einen Künstlernamen handelt, der auf eine Rolle zurückgeht, die die Sängerin in einem musikdramatischen Werk verkörpert hatte.

Schließlich enthält ein Bericht, den Pio im November 1687 dem Kaiser zukommen ließ, eine Beilage mit Nachrichten aus Turin, Genua, Madrid, Mailand, Rom und Venedig, wobei in den Nachrichten aus Turin von den Sängerinnen „Clarice“ und „Barbaruccia“ sowie vom Sänger „Cortona“ die Rede ist. Laut Berichterstatter wurden alle drei engagiert, um in einer musikalischen Darbietung zu singen, die im Hause eines gewissen „General Grondana“ angesetzt war (Anhang VIII.16). Dieser „General“ ist als Gian Matteo Grondana, Mitglied des savoyischen Hofstaats, zu identifizieren. Bei den engagierten Sängerinnen dürfte es sich um Clarice Gigli aus Florenz und um Barbara Riccioni, die auch „la Romanina“ genannt wurde, handeln. Während beide im Laufe der Zeit weitgehend in Vergessenheit gerieten, ist der als „il Cortona“ bekannte Soprankastrat Domenico Cecchi heute zumindest in Fachkreisen kein unbeschriebenes Blatt. Cecchi durchlief eine große internationale Musikerkarriere, zu deren zahlreichen Stationen auch Wien zählte, wo der Sänger wiederholt Dienste für die Habsburger leistete, zuletzt als Musiklehrer der Erzherzoginnen während der Regentschaft Josephs I. ${ }^{38}$

35 Der damalige sizilianische Vizekönig Francisco IV. de Benavides y Dávila war Vater zahlreicher Kinder.

36 Vgl. GLÜXAM, Dagmar - FASTL, Christian. Landini-Contini (Landini-Conti, La Conti, La Contini, La Landina, di Chateauneuf, detta Landini, di Castelnuovo), Maria. In OeMl, online. [cit. 2018-01-18]. URL: http://www.musiklexikon.ac.at/ml/musik_L/Landini-Contini_Maria.xml. ZILLI, Anna. Christina of Sweden queen of music in Rome and the women singers at her service. Il Ganassi. Bollettino della Fondazione Italiana per la Musica Antica, 2014, vol. 17, no. 14, S. 2-4.

37 Vgl. SCARLATTI, Alessandro. Solo serenatas. Marie-Louise Catsalis - Rosalind Halton (Hg.). Middleton, Wis.: A-R Ed., 2011, S. XV, XX.

38 Vgl. SEIFERT, Nordwärts reisende Gesangsvirtuosen, op. cit., S. 169. Riccioni und Cecchi standen gleichzeitig im Dienst des Herzogs von Mantua und traten immer wieder gemeinsam auf, u. a. auch in Rom. Siehe DELLA LIBERA, Luca - DOMÍNGUEZ, José María. Nuove fonti per la vita musicale romana di fine Seicento: il Giornale e il Diario di Roma del Fondo Bolognetti all'Archivio Segreto Vaticano. In La Musique à Rome au XVII siècle. Études et perspectives de recherche. Caroline Giron-Panel - Anne-Madeleine Goulet. Rom: École française de Rome, 2012, S. 160. 


\section{Anhang}

\section{Haus-, Hof- und Staatsarchiv, Diplomatie und Außenpolitik, Rom}

\section{Korrespondenz 54}

Sac. Ces. ${ }^{a}$ Real Maestà mio Sig. clem. ${ }^{\text {mo }}$

La clem. ${ }^{\text {ma }}$ di V. M. delli 8. Marzo mi è capitata quà alli 2. del corrente, onde non posso assistere à D. Giacinto Cornaccioli nelli affari comessili dalla M. V., e nelli interessi particolari di lui come benigniss. ${ }^{\text {te }}$ mi ordina, e farò al mio ritorno in Roma se sarà in tempo. E non havendo cosa aggiongere con $\mathrm{q}[\mathrm{ue}] \mathrm{sta}$ ne di nuovo, ne di negotio, resto inchinando umiliss. ${ }^{\text {te }}$ V. M. cui Dio concedi longhiss. ${ }^{i}$ e gloriosiss. ${ }^{i}$ anni di vita. Di Bozolo 5. Giugno 1640.

Di V[ost]ra Sac. Ces. ${ }^{a}$ Real Maestà

Umiliss. ${ }^{\circ}$ divotiss. $^{\circ}$ e fedeliss. ${ }^{\circ}$ vassallo e serv. ${ }^{\text {re }}$

Scip[ion]e Duca di Sab[bione]ta

\section{Korrespondenz 56}

\section{Sacra Cesarea Maestà}

Parlai à sua Santità fin dall'audienza passata del particolare da V. M. ${ }^{\text {tà }}$ Ces. ${ }^{a}$ benignam. te commessomi colla clement. ${ }^{\text {ma }}$ sua de 15 . di Marzo per Pietro Giorgio Picolini Musico già di questa Cappella Pontificia, mà ritrovando in sua Sant. ${ }^{\grave{a}}$ puoco buona dispositione verso di lui, per il mal procedere, e forma di vita ch'egli haveva tenuto per il passato, hò voluto prima di rispondere aspettar' il rescritto, che erà uscito al memoriale, ch'io ne presentai alla Sant. ̀̀ sua, qual' è che à suo tempo, se ne sarebbe tenuto memorià. Ch'è quanto per hora succede in questo particolare, et à V. M. Ces. ${ }^{a}$ faccio hum. ${ }^{\text {ma }}$ riverenza. Albano 28. Aprile 1646.

D. V. M. ${ }^{\text {tà }}$ Cesarea

Humilss. ${ }^{\text {mo }}$ et obligat. ${ }^{\text {mo }}$ Serv. ${ }^{\text {re }}$ vero

Federico Savelli

\section{Korrespondenz 56}

\section{Sacra Maestà Cesarea}

Da Marc' Antonio Ferri Musico di Camera di V. M. Ces. a , mi è stata ultimamente resa la clement. ${ }^{\text {ma }}$ sua delli 15. di luglio passato, à favor' di lui medesimo. Dall'istesso ancora V. M. Ces. ${ }^{a}$, si degnerà d'intendere, quanto volentieri l'habbi eshibito l'opera mia in tutte l'occorrenze, ch'egli mi porgerà di suo servitio, stimando cosi dovuto alli benignissimi cenni di V. M. Ces. ${ }^{\text {a }}$, e alle persone, che dipendono dal suo Imperial serv. ${ }^{\circ}$ E le faccio humilissima riverenza. Roma li 18. settembre 1649.

D. V. M. ${ }^{\text {tà }}$ Cesarea

Humilss. ${ }^{\text {mo }}$ et obligat. ${ }^{\text {mo }}$ Serv. ${ }^{\text {re }}$ vero

Federico Savelli 


\section{Varia 10}

Alla Sacra Cesarea Maestà ecc. / Humiliss. ${ }^{\text {ma }}$ Supp[lic]a per / Antimo Liberati per una Lettera di Raccomandatione.

Sacra Cesarea Maesta Sig. ${ }^{\text {re }}$ Sig. ${ }^{\text {re }}$ Sempre Clement. ${ }^{\text {mo }}$

Antimo Liberati già humiliss. ${ }^{\text {mo }}$ Servo della Augustiss. ${ }^{\text {ma }}$ Casa supp[li]ca humilissimam. te la M. ${ }^{\text {ta }} \mathrm{V} \cdot{ }^{\text {ra }}$ che vacando un luoco di Contralto nella Capella ponteficia si degni p[er] sua Clemenza favorirlo di una Lett. ${ }^{a}$ di efficace raccomanda[tione] con Sigillo piccolo All'Eminent. ${ }^{\text {mo }}$ Cardinal d'Harrach ò All'Eminent. ${ }^{\text {mo }}$ Cardinal Landgraino acciò in voce, l'intercedano dal nuovo Pontefice il sop. ${ }^{a} \mathrm{~d}[\mathrm{ett}]$ o loco in Capp. ${ }^{a}$ ò altro con titolo di sopranumerario conforme con infeniti altri è seguito; che cosi si riempirà il numero preciso delle voci. Il Pontefice acquisterà senza pregiuditio, ne spesa della Camera un Servitore habile, ed idoneo, come in occasione di concorso è stato approvato dal Collegio p[er] suoi Musici e la M. ${ }^{\text {ta }} \mathrm{V}^{\text {ra }}$ haverà un perpetuo servo in Roma, che non solo raguaglierà di quelli Musici che la M. ${ }^{\text {ta }}$ vostra vorrà servirsi in Avenire, ma sarà pronto ancora ad impiegarsi in qualsivoglia cosa che di ordine di V. M. ${ }^{\text {ta }}$ li sara ordinato, mentre sperando ottenere la gratia profondam. ${ }^{\text {te }}$ Alla $M .{ }^{\text {ta }} \mathrm{V} \cdot{ }^{\mathrm{a}}$ se inchina.

\section{Korrespondenz 59}

17. Febr. 1655.

Card[inali] ab Harrah pro Antimo Librati [sic] musico.

Ferd[inandus] 3 .

Ex incluso supplicis libelli exemplo videbit R[everendissi]ma V[estra] P[aterni]tas, quem locum Contralti in Pontificia Capella sibi concedi roget Antimus Librati, quom si commodi fieri possit, voti compotem reddi cupientes, a R[everendissi]ma V[estra] P[aternita]te clement[iam] postulamus, ut si quidem effectum commendationis $\mathrm{N}$ [ost]rae secuturum videat, supplicanti no[min]e $\mathrm{n}[\mathrm{ost}]$ ro favorem suum in obtinendo desiderio impertiatur: Qui R[everendissi]mae P[aternita]ti V[estrae] gra[ti]ae et benevol[ent]iae $\mathrm{N}$ [ost]rae affectum conservamus.

Viennae 17. feb. [1]655

\section{Varia 10}

Alla Sac. ${ }^{\text {a }}$ Ces. ${ }^{\text {a }}$ M..$^{\text {tà }}$ dell'Imperatore, $S .{ }^{\text {re }}$, S. $^{\text {re }}$ sempre Clementiss. ${ }^{\circ} / 11$. Julij $1655 / \mathrm{Hu}-$ miliss. ${ }^{a}$ Supplica di / Antimo Liberati musico in Roma. Per una Lettera di raccomandatione.

Sac: Ces. ${ }^{\text {a }} \mathrm{M} .{ }^{\text {tà }}, \mathrm{S}^{\text {re }},{ },{ }^{\text {re }}$ sempre Cleme[n]tiss. $^{\circ}$

Antimo Liberati, già humiliss. ${ }^{\circ}$ servo nella Musica dell'Augustiss. ${ }^{a}$ Casa, supplica humiliss. ${ }^{\text {te }}$ la benigna clemenza della M. V. degnarsi honorarlo di una lettera con sigillo picciolo di efficace raccomandatione all'Eminentiss. ${ }^{\circ}$ S. ${ }^{r}$ Cardinal Gio: Carlo de Medici, acciò gl'interceda dalla Santità del Papa un loco musica di sopranumerario nella Cappella Pontificia; che essendo grazia solita à concedersi, tiene di certo doverla co[n]seguire, mediante la potentiss. ${ }^{a}$ intercessione della M. V., alla quale restarà perpetuame[n]te obligato, e di mostrarsi sempre pronto à suoi gratiosiss. ${ }^{i}$ comandi, e di pregare Iddio per la conservazione della sua Sacra Cesarea persona. Quam Deus. 
Di V. Sac. Ces. M.tà

Humiliss. ${ }^{\circ}$, e Devotiss. ${ }^{\circ}$ Servo.

Antimo Liberati.

\section{Hofkorrespondenz 13}

\section{Aug. 1655}

Card. ${ }^{\mathrm{li}}$ Io: Carolo de Medices pro musico Antimo Liberati

Ferd[inandus] $3^{\text {tius }}$

Antimus Librati, qui nobis operam suam aliquandiu in choro $\mathrm{N}$ [ostr] o musico probavit, id vehementer exoptat, ut locum aliquem supernumerarium in Capella Pontificia ad $\mathrm{N}$ [ost]ram interpo[sition]em consequi possit, et cum eiusmodi gratia aliis quoquenti nobis refertur, concedi soleat. Tanto libentins supplicantis precibus annuendum atque $\mathrm{R}$ [everendissi]mam $\mathrm{P}$ [aternita]tem $\mathrm{V}[$ estram] hisce amice ac benevole requirendam duximus, quatenus apud S[anctita]tem Suam n[ost]ro no[min]e officia sua interponere non gravitur, quo p[ro]fatus musicus desideratum locu[m] in Capella obtineat, adeoque commendationis N[ostr]ae fructum ab effectu experiatur: Id Nobis gratum erit, qui R[everendissi]mae $\mathrm{P}[$ aternita]ti $\mathrm{V}$ [estrae] benevol[ent]iae $\mathrm{N}[$ ost]rae affectu[m] conservamus. Eberstorffii, 2. Aug. [1]655. ${ }^{39}$

\section{Kardinal Carlo Pio di Savoia an Kaiser Leopold I.}

\section{Korrespondenz 60}

Rom, 24. Oktober 1676

Riservato.

Alesandro Stradella Compositor di Musica havendo indotto un certo Innocenzio Cibo di natura scemo à far pri. ${ }^{\text {ma }}$ donaz. ${ }^{\text {ne }}$ di tutto il suo ad una Donna detta la Pescatora e dopo a sposarla, procurò seguisse il Matrimonio segretam. ${ }^{\text {te }}$ in Casa del Sig. ${ }^{\mathrm{r}}$ Duca di Bracciano nelle stanze di un Musico di Cappella suo amico serv[ito]re attuale di quella Casa, il S. ${ }^{\mathrm{r}}$ Card. ${ }^{1}$ Cibo mandò dal Sig. ${ }^{\mathrm{r}}$ Duca di Bracciano a dirle che voleva fosse la Donna inviata nel Conservatorio di Monte Citorio e dopo fù il Giudice del Vicario ad esaminare ambedue.

\section{Korrespondenz 61}

Rom, 5. Februar 1678

Giovedi sera fù recitata l'opera in Musica della Baronessa cantata da diversi Cavalieri, e domani a sera si rappresenterà quella di Mattia de Rossi.

\section{Korrespondenz 61}

Rom, 12. Februar 1678

La Regina di Svezia è intervenuta due volte con alcuni Sig. ${ }^{\text {ri }}$ Cardinali e Prelati alla Comedia di Antonio Masini, rappresentata in Casa di Monsù Ridò a Monte Brianzo.

Si è recitata più volte in questa settimana quella della Baronessa, come anche quella di

39 Der Entwurf wurde im Nachhinein von anderer Hand korrigiert. Die Korrekturen sind in der Transkription nicht berücksichtigt. 
Mattia de Rossi, e molte altre Comedie di particolari che trà tutte si fà conto passino il numero di cinquanta.

\section{Korrespondenz 64}

Rom, 17. Juli 1683

Riservato.

Fece dire l'Amb[asciato]r di Francia à Gio. Francesco Musico chiamato comunem[en]te col nome di Siface, che voleva cantasse la sera. Rispose questi esser stuffo di cantare à uffo, e tacciando di scrocco l'Amb[asciato]re p[er] non haverli mai donato cosa alcuna benche molte volte l'havesse fatto cantare, con altri termini poco riverenti ricusò di servirlo. L'Amb[asciato]re alterato fece minacciarlo di bostone, disse al Card. Maidalchino che lo scacciasse di sua Casa, dove egli abitava, se ne andò nel Palazzo del Duca di Modona, al q[ua]le serve, e si sente che dopo se ne sia partito.

\section{Korrespondenz 64}

Rom, 4. März 1684

Di mal di volvolo è passato all'altra vita Francesco Maria Fede Musico celebre, per la di cui morte vaca un Luogo nella Cappella Pontificia.

\section{Korrespondenz 64}

Rom, 12. August 1684

Domenica sera fù fatta da questi S[igno]ri Buzij una Serenata nella piazza del Gesù, la quale non hebbe alcun applauso da glie astanti.

Altra Serenata fù fatta Giovedi a sera con molti Instrumenti alla M[ae]stà della Regina di Svezia, nella quale cantò il Ballarino Contralto del Ser. ${ }^{\mathrm{mo}}$ S. ${ }^{\mathrm{r}}$ Duca di Mantova.

\section{Korrespondenz 64}

Rom, 23. September 1684

La Baronessa famosa Cantatrice in questa Città, havendo fatta la risoluzione d'entrare in Monastero, et eletto quello di Sant'Ambrogio vi fù condotta dalla Sig. ${ }^{\text {ra }}$ Duchessa di Bracciano. Dopo il suo ingresso, le Monarche pentite d'haverla accettata, fecero ricorso a S. Beat. ${ }^{\text {ne }}$ p[er]che n'uscisse, come segui con grave sentimento della Duchessa pred[ett] a, dalla quale fù messa in deposito nelle Zitelle di S[a]nta Eufemia per fin’ à tanto le trovi altro Monastero che la riceva.

\section{Korrespondenz 64}

Rom, 2. Dezember 1684

Riservato.

Con l'occasione entrò nelle monache di Sant'Ambrosio la Baronessa famosa cantatrice, benche $\mathrm{p}[\mathrm{er}]$ monacarsi, parte di quelle monache, che non la volevano, ricorsero al Papa $\mathrm{p}$ [er]che la facesse uscir subito p[er] esser magg[io]re di 25. anni. Diede S[ua] B[eatitudi] ne l'ordine desiderato $\mathrm{p}$ [er] l'espulsione della Baronessa, che segui, mà ingiunse anche il comando al S. ${ }^{\mathrm{r}}$ Card. Vicario di fare uscire dalli monasterij tutte quelle erano arrivate 
alli 25 anni senz'haver preso stato conforme gli ordini della Cong[regazio]ne de' Vescovi non mai sin'ora esseguiti. Il card. Vicario andò differendo l'esecuz[io]ne, avedutosene il Papa mandò un'ordine sotto li 15. di novembre à tutti li monasterij che facessero uscire in termine d'un mese tutte l'educande che arrivavano alli 25. anni sotto pena della scomunica ipso facto incurrenda. Molte sono le giovani che dovranno uscire tenute p[er] forza sin'ora, ò dalla poca carità de' parenti, ò dell'impossibilità di far la spesa p[er] monacarle.

\section{Korrespondenz 65}

Rom, 20. Oktober 1685

Per la morte di Giovan Ricchi Musico di Cappella vacò un Cannonicato alla Rotonda, quale fù dato il giorno segu[en]te ad un Prete Segretario del S. ${ }^{\text {re }} \mathrm{D}$ [on] Livio [Odescalchi].

\section{Korrespondenz 65}

Rom, 25. Mai 1686

Riservato.

Essendo stata avisata la Giorgina famosa Canterina che S. B. ${ }^{\text {ne }}$ voleva farla porre in un Conservatorio $\mathrm{p}$ [er] dubio che non fosse andata à Mantova, $\mathrm{p}$ [er] non $\mathrm{p}$ [er]dere la sua libertà andò à racomandarsi alla Regina di Svezia sotto la di cui prottez[io]ne ella vive, quale fece trattenerla nel suo Palazzo, ove de presente dimora.

\section{Korrespondenz 65}

Rom, 27. Juli 1686

Mercordi a sera fu fatta una Serenata sopra la Ringhiera del Cortile della Regina di Svezia, nella q[ua]le cantorono la Giorgina famosa Cantatrice al servizio di S. M[ae]stà, e la figlia del suo Capitano della Piazza, vi concorso gran num. ${ }^{\circ}$ di gente e nobiltà a goderne.

\section{Korrespondenz 66}

Rom, 4. Januar 1687

Giunse qui il March. ${ }^{\mathrm{e}}$ di Solera figlio del S. Co: di San Stefano V[ice]rè di Sicilia, al q[ua] le fù dato una di q[ue]ste sere dal March. ${ }^{e}$ del Monte il divertim[en]to d'una Conversaz[io]ne di Dame, nella quale cantò la Giorgina famosa Cantatrice.

\section{Korrespondenz 66}

Rom, 19. April 1687

Dal Sig. ${ }^{r}$ Cardinal de Medici si canta frequentemente la sera, dove concorrono molti $\mathrm{p}$ [er] sentire il famoso Musico Vincenzino.

Anche dalla Regina di Svezia fù fatta Dom. ${ }^{\text {ca }}$ sera un'Accademia di Musici forestieri, e di quelli di Roma, dove intervennero diversi Prelati e Cav. ${ }^{\text {ri }}$ di questa Città.

\section{Korrespondenz 66}

Rom, 26. April 1687 
Domenica sera furono fatte alla Trinità de Monti le allegrezze de fuochi $\mathrm{p}$ [er] la ricuperata salute del Rè Xmo, [...]

Anche l'Abbate Elpidio Benedetti fece la sera seguente le sue dimostrazioni d'allegrezza con l'illuminazione nella facciata della sua Casa, dove intervennero la Sig. ${ }^{\text {ra }}$ Duchessa di Bracciano, et il Sig. ${ }^{r}$ Card. ${ }^{1}$ d'Etrè [= César d'Estrées], e fece cantare sopra l'Arringhiera della medesima una Cantarina detta la Capitaniessa, che trasse maggior la curiosità del Popolo in quel Luogo.

\section{Korrespondenz 66}

Rom, 21. Juni 1687

Lunedi passato [...] Nella med. ${ }^{\text {ma }}$ sera fù fatta dalla Reg[in]a di Svezia un'Academia di Musica, alla quale intervenne il famoso Musico Vincenzino, ed altri Musici forestieri.

\section{Korrespondenz 66}

Turin, 1. November 1687

[...] et è giunta da Firenze la Clarice Cantatrice $\mathrm{p}[\mathrm{er}]$ recitare nell'opera musicale alloggiata in Casa del G[e]n[er]al Grondana, e devono venir p[er] lo stesso fine la Barbaruccia Romana, et il Cortona.

\section{Korrespondenz 66}

Rom, 10. Juli 1688

Sopra la Ringhiera del Palazzo della Reg. ${ }^{a}$ di Svezia fù Martedi a sera fatta una Serenata, nella quale cantarono la Giorgina, e la figlia del Capitan Landini virtuosa di S. M[ae]stà. Vi concorso molti Cav. ${ }^{\text {ri }}$ e Dame à goderne, e fra queste la Sig. ${ }^{\text {ra }}$ Duchessa di San Pietro.

\section{Bibliography}

ADAMI, Andrea. Osservazioni per ben regolare il coro de i cantori della Cappella Pontificia. Rom: Antonio de' Rossi alla Piazza di Ceri, 1711.

ANTONICEK, Theophil. „Pigliar musici dall’ Italia“. Ein Agent des Erzherzogs Leopold Wilhelm auf der Suche nach italienischen Musikern: Giacinto Cornacchioli. Studien zur Musikwissenschaft, 2017, S. 7-60.

BORCHIA, Matteo. Gli agenti delle corti tedesche a Roma nel XVIII secolo. Dissertation. Rom 20102011.

CILIBERTI, Galliano. Antonio Maria Abbatini e la musica del suo tempo (1595-1679). Documenti per una ricostruzione bio-bibliografica. Perugia: Gestisa, 1986.

Convegno Internazionale. Cristina di Svezia e la Musica (Roma, 5-6 dicembre 1996). Rom: Accademia Nazionale dei Lincei, 1998.

DIXON, Graham. Lorenzo Ratti (1589/90-1630): “Exemplum Virtutum”. Note d'Archivio per la Storia Musicale, 1984, vol. 2, S. 7-20.

FERRAROTTI, Mario. Pietro Giorgio Picciolini: Basso vercellese. Vercelli: De Marchi, 1946.

GIANTURCO, Carolyn. Stradella. «uomo di gran grido». Pisa: Edizioni ETS, 2007. 
HEYINK, Rainer. Fest und Musik als Mittel kaiserlicher Machtpolitik. Das Haus Habsburg und die deutsche Nationalkirche in Rom S. Maria dell'Anima. Tutzing: Schneider, 2010.

MORELLI, Giorgio. Una celebre "Canterina" romana del Seicento, La Giorgina. Studi Secenteschi, 1975, vol. 16, S. 157-180.

NEVEU, Bruno. Episcopus et Princeps Urbis: Innocent XI réformateur de Rome d'après des documents inédits (1676-1689). In Römische Kurie. Kirchliche Finanzen. Vatikanische Archive. Studien zu Ehren von Hermann Hoberg, vol. 2. Erwin Gatz (Hg.). Rom: Università Gregoriana Editrice, 1979, S. 597-633.

PRZYBYSZEWSKA-JARMIŃSKA, Barbara. Muzyczne kontakty dworu królewskiego polskich Wazów z Rzymem w świetle dawniejszych i nowych badań. De Musica / Diagonali, 2012, vol. 1, S. $1-28$.

RAMBOTTI, Fiorella. «La musica è una mera opinione e di questa non si può dar certezza veruna» Antimo Liberati e il suo Diario sistino con una riproduzione della Lettera a Ovidio Persapegi. Perugia: Morlacchi, 2008.

ROSTIROLLA, Giancarlo. Celebrazioni ed eventi musicali nella Cappella Pontificia durante l'Anno Santo 1625: Dal Diario redatto dal compositore e cantore perugino Francesco Severi, con l'edizione integrale di esso. In »Vanitatis fuga, aeternitatis amor«. Wolfgang Witzenmann zum 65. Geburtstag. Laaber: Laaber-Verlag, 2005, S. 151-226.

SEIFERT, Herbert. Texte zur Musikdramatik im 17. und 18. Jahrhundert. Aufsätze und Vorträge. Matthias J. Pernerstorfer (Hg.). Wien: Hollitzer, 2014.

STAFFIERI, Gloria. Colligite Fragmenta. La vita musicale romana negli «Avvisi Marescotti» (16831707). Rom: Libreria musicale italiana editrice, 1990.

TAMBURINI, Elena. La lira, la poesia, la voce e il teatro musicale del Seicento: note su alcune vicende biografiche e artistiche della baronessa Anna Rosalia Carusi. In La musica a Roma attraverso le fonti d'archivio. Atti del convegno internazionale, Roma 4-7 giugno 1992. Bianca Maria Antolini - Arnaldo Morelli - Vera Vita Spagnuolo (Hg.). Lucca: Libreria Musicale Italiana, 1994, S. 419-432.

ZILLI, Anna. Cristina di Svezia regina della musica a Roma. Le "canterine" al suo servizio. Rom: Aracne, 2013. 